\title{
Comunicación
}

\section{Diagnóstico Histopatológico de Neoplasias en Tracto Reproductivo de Caninos y Felinos Hembras Realizadas en el Laboratorio de Patología Animal de la Universidad Nacional Mayor de San Marcos (2007-2015)}

\author{
Histopathology Diagnosis of Neoplasms in the Reproductive Tract of Female \\ Dogs and Cats at the Animal Pathology Laboratory of \\ SAn Marcos University (2007-2015) \\ Daniel Salazar C. ${ }^{1}$, Rosa Perales C., ${ }^{1,2}$
}

\section{Resumen}

El objetivo del presente estudio retrospectivo fue determinar la frecuencia de neoplasias del aparato reproductivo de caninos y felinos hembras según los registros histopatológicos del Laboratorio de Patología Animal de la Universidad Nacional Mayor de San Marcos (periodo 2007-2015), evaluando datos sobre raza, edad, localización anatómica y diagnóstico histopatológico. La frecuencia de neoplasias del aparato reproductor de hembras caninos fue de $5.99 \pm 1.16 \% \mathrm{IC}_{95 \%}(96 / 1603)$ y para felinos fue de $4.41 \pm 4.88 \%$ $\mathrm{IC}_{95 \%}(3 / 68)$. En caninos, la mayor frecuencia se presentó en razas puras $(60.22 \pm 9.95 \%)$, en canes entre 8 a 12 años $(43.75 \pm 9.92 \%)$ y mayormente en la zona vulvar (37.50 $\pm 9.68 \%)$. La neoplasia más frecuente en el ovario fue el tumor de células de la granulosa $(47.6 \pm$ $21.4 \%)$, en útero fue el leiomioma $(50.0 \pm 40.0 \%)$ y en vulva y vagina fue el tumor venéreo transmisible $(39.4 \pm 16.7 \%)$. En felinos se encontraron dos disgerminomas en ovarios y un fibroadenoma polipoide en útero.

Palabras clave: tracto reproductor femenino, neoplasias, frecuencia, canino, felino

\section{AbSTRACT}

The aim of this retrospective study was to determine the frequency of tumors in the reproductive organs of female canines and felines, based on histopathological records of the Animal Pathology Laboratory of the National University of San Marcos (period 20072015), and to correlate breed, age and anatomical site with the histopathological diagno-

\footnotetext{
${ }^{1}$ Laboratorio de Histología, Embriología y Patología Animal, Facultad de Medicina Veterinaria, Universidad Nacional Mayor de San Marcos, Lima, Perú

${ }^{2}$ E-mail: rperales_fmv@hotmail.com
} 
sis. The frequency of neoplasia in the canine reproductive organs was $5.99 \pm 1.16 \% \mathrm{IC}_{95 \%}$ and in feline was $4.41 \pm 4.88 \%$. In bitches, tumors were most often found in pure breeds $(60.22 \pm 9.95 \%)$, between 8 to 12 years $(43.75 \pm 9.92 \%)$ and mostly in the vulvar area $(37.50$ $\pm 9.68)$. The most common tumor in the ovary was the tumor of granulosa cells (47.6 \pm $21.4 \%)$, while in uterus was leiomyoma $(50.0 \pm 40.0 \%)$ and in the vulva and vagina was the transmissible venereal tumor $(39.4 \pm 16.7 \%)$. In cats two dysgerminomas in ovaries and one polypoid fibro-adenoma in uterus was found.

Key words: female reproductive tract, neoplasms, frequency, canine, feline

\section{INTRODUCCIÓN}

Las neoplasias del tracto reproductor femenino presentan una baja frecuencia respecto al total de neoplasias en caninos y felinos debido a la cultura de esterilizar a temprana edad o no llegar a un diagnóstico definitivo del tumor al ser hallazgos accidentales en cirugías (McEntee y Nielsen, 1976; González et al., 1997; Sforna et al., 2003). Estas neoplasias pueden ser benignas o malignas y desarrollarse a nivel ovárico, uterino, vaginal y vulvar.

Las neoplasias ováricas representan entre el 0.5 a $1.2 \%$ y el 0.7 a $3.6 \%$ sobre el total de neoplasias en perras y gatas, respectivamente (Klein, 2007). Se reportan neoplasias desde los 14 meses hasta los 16 años de edad en perras, y en gatas desde los 2 meses hasta los 20 años de edad (Hermo et al., 2010). Las neoplasias pueden ser clasificadas como epiteliales, del estroma de células sexuales y de células germinales. Las neoplasias de tejido de sostén y metastásicas son infrecuentes en caninos y felinos, siendo los hemangiomas y leiomiomas los de mayor presentación (Jubb et al., 1991; Baba y Catoi, 2007).

Las neoplasias uterinas representan del 0.3 al $0.4 \%$ del total de neoplasias en caninos y de 0.2 a $1.5 \%$ en felinos (Klein, 2007; Hermo et al., 2010), siendo más frecuente la presentación de tumores de carácter benigno en perras y maligno en gatas (Morris y Dobson, 2001; Dobson y Lascelles, 2010). E1 leiomioma es la neoplasia benigna más común en perras (85-90\%), y el leiomiosarcoma es el tumor maligno más frecuente $(10 \%)$ frente al total de neoplasias uterinas (Hermo et al., 2010; Thomson y Britt, 2012). En el caso de felinos, el adenocarcinoma es la neoplasia maligna de mayor frecuencia (Miller et al., 2003).

Las neoplasias vaginales y vulvares ocupan el segundo lugar en incidencia de tumores del tracto reproductor en hembras caninas, seguidos de las neoplasias mamarias (Baba y Catoi, 2007; Hermo et al., 2010; Thomson y Britt, 2012). Las neoplasias benignas de músculo liso son las más reportadas (80-90\%), en tanto que a nivel de los labios vulvares se puede apreciar todo tipo de neoplasias cutáneas. En el caso de felinos, las neoplasias vaginales y vulvares son raras (Morris y Dobson, 2001; Dobson y Lascelles, 2010). En el caso de las neoplasias epiteliales, su frecuencia es mucho menor que las neoplasias mesenquimales, habiéndose descrito papilomas y carcinomas, siendo el carcinoma de células escamosas el más frecuente (McEntee y Nielsen, 1976).

Una neoplasia que no entra en la clasificación de mesenquimal o epitelial y es bastante frecuente en el país a nivel de vulva y vagina de caninos es el tumor venéreo transmisible (TVT). Esta neoplasia se transmite a través del coito desde animales infectados a animales sanos, mostrando una locación genital o extragenital (Das y Das, 2000; Mello et al., 2005; Mendoza, 2006). 
Cuadro 1. Distribución de neoplasias del tracto reproductor femenino según raza, edad y localización anatómica en caninos con base a diagnósticos histopatológicos en el Laboratorio de Patología de la UNMSM (2007-2015)

\begin{tabular}{|c|c|c|c|}
\hline \multicolumn{2}{|l|}{ Variables } & \multirow{2}{*}{$\begin{array}{c}\text { Casos } \\
(\mathrm{n}) \\
56\end{array}$} & \multirow{2}{*}{$\begin{array}{c}\text { Frecuencia } \\
\left(\% \pm \text { IC }_{95 \%}\right)\end{array}$} \\
\hline Raza & Pura & & \\
\hline & Cruzada & 37 & $39.78 \pm 9.95$ \\
\hline \multirow[t]{5}{*}{ Estrato etario (años) } & $0-3$ & 8 & $8.33 \pm 5.53$ \\
\hline & $4-7$ & 33 & $34.38 \pm 9.50$ \\
\hline & $8-12$ & 42 & $43.75 \pm 9.92$ \\
\hline & $>12$ & 11 & $11.46 \pm 6.37$ \\
\hline & No registra & 2 & $2.08 \pm 2.85$ \\
\hline \multirow[t]{4}{*}{ Localización anatómica } & Ovario & 21 & $21.88 \pm 8.27$ \\
\hline & Útero & 6 & $6.25 \pm 4.84$ \\
\hline & Vagina & 33 & $34.38 \pm 9.50$ \\
\hline & Vulva & 36 & $37.50 \pm 9.68$ \\
\hline
\end{tabular}

El objetivo del presente estudio fue determinar la frecuencia de neoplasias del tracto reproductor femenino en caninos y felinos, con base a registros del laboratorio de patología animal de la Universidad Nacional Mayor de San Marcos del periodo 2007-2015.

\section{Materiales y MéTodos}

El presente estudio se realizó en el Laboratorio de Histología, Embriología y Patología Animal de la Facultad de Medicina Veterinaria, Universidad Nacional Mayor de San Marcos (LHEPA-FMV-UNMSM). Se revisaron los informes histopatológicos correspondientes a las especies canina y felina con diagnóstico de neoplasia en el tracto reproductor femenino en el periodo comprendido entre el 1 de enero de 2007 al 31 de diciembre de 2015.

Las variables sometidas al estudio fueron raza (pura, cruzada), edad (0-3, 4-7, 8-
$12,>12$ años), localización anatómica (ovario, útero, vagina, vulva) y diagnóstico histopatológico.

\section{Resultados}

Se dispuso de 1603 registros de casos diagnosticados histopatológicamente como neoplasias en caninos. De estos, 96 casos afectaron al tracto reproductor femenino, representando el $5.99 \pm 1.16 \% \mathrm{IC}_{95 \%}$. Asimismo, fueron diagnosticados 68 casos de neoplasias en felinos, donde tres casos afectaron al tracto reproductor femenino, siendo una frecuencia de $4.41 \pm 4.88 \% \mathrm{IC}_{95 \%}$.

La frecuencia de distribución de neoplasias del tracto reproductor femenino, según raza, edad y localización anatómica en caninos se presenta en el Cuadro 1. Se observó una mayor frecuencia en razas puras $(60.22 \% ; 56 / 93)$ frente a canes cruzados (39.78\%; 37/93). Dentro de las razas puras, 
Cuadro 2. Frecuencia de neoplasias del tracto reproductor femenino en canes según Ubicación anatómica y diagnóstico histopatológico en el Laboratorio de Patología de la UNMSM (2007-2015)

\begin{tabular}{|c|c|c|c|}
\hline $\begin{array}{c}\text { Localización } \\
\text { anatómica }\end{array}$ & Diagnóstico histopatológico & $\begin{array}{c}\text { Casos } \\
\left(\mathrm{N}^{\circ}{ }^{\circ}\right)\end{array}$ & $\begin{array}{l}\text { Frecuencia por } \\
\text { órgano }(\% \pm \\
\left.\mathrm{ICc}_{95 \%}\right)\end{array}$ \\
\hline $\begin{array}{l}\text { Ovario } \\
\text { (21) }\end{array}$ & $\begin{array}{l}\text { Tumor de las células de la granulosa } \\
\text { Adenocarcinoma papilar } \\
\text { Disgerminoma } \\
\text { Tumores mixtos } \\
\text { Teratoma } \\
\text { Hemangiosarcoma } \\
\text { Leiomioma ovárico } \\
\text { Adenocarcinoma cistopapilar } \\
\text { Tecoma }\end{array}$ & $\begin{array}{l}10 \\
2 \\
2 \\
2 \\
1 \\
1 \\
1 \\
1 \\
1\end{array}$ & $\begin{array}{l}47.6 \pm 21.4 \\
9.5 \pm 12.5 \\
9.5 \pm 12.5 \\
9.5 \pm 12.5 \\
4.8 \pm 9.1 \\
4.8 \pm 9.1 \\
4.8 \pm 9.1 \\
4.8 \pm 9.1 \\
4.8 \pm 9.1\end{array}$ \\
\hline $\begin{array}{l}\text { Útero } \\
(6)\end{array}$ & $\begin{array}{l}\text { Leiomioma } \\
\text { Leiomiosarcoma } \\
\text { Adenocarcinoma angiomatoso } \\
\text { Fibrosarcoma }\end{array}$ & $\begin{array}{l}3 \\
1 \\
1 \\
1\end{array}$ & $\begin{array}{l}50.0 \pm 40.0 \\
16.7 \pm 29.8 \\
16.7 \pm 29.8 \\
16.7 \pm 29.8\end{array}$ \\
\hline $\begin{array}{l}\text { Vagina } \\
(33)\end{array}$ & $\begin{array}{l}\text { TVT } \\
\text { Fibroma } \\
\text { Fibrosarcoma } \\
\text { Rabdomiosarcoma } \\
\text { Fibroleiomioma } \\
\text { Carcinoma escamoso } \\
\text { Hemangiosarcoma } \\
\text { Hemangioma } \\
\text { Rabdomioma } \\
\text { Mastocitoma } \\
\text { Mixofibroma angiomatoso (fibroma molle) } \\
\text { Fibroleiomiosarcoma } \\
\text { Lipoma } \\
\text { Linfosarcoma }\end{array}$ & $\begin{array}{l}13 \\
3 \\
3 \\
2 \\
2 \\
2 \\
1 \\
1 \\
1 \\
1 \\
1 \\
1 \\
1 \\
1\end{array}$ & $\begin{aligned} 39.4 & \pm 16.7 \\
9.1 & \pm 9.8 \\
9.1 & \pm 9.8 \\
6.1 & \pm 8.1 \\
6.1 & \pm 8.1 \\
6.1 & \pm 8.1 \\
3.0 & \pm 5.9 \\
3.0 & \pm 5.9 \\
3.0 & \pm 5.9 \\
3.0 & \pm 5.9 \\
3.0 & \pm 5.9 \\
3.0 & \pm 5.9 \\
3.0 & \pm 5.9 \\
3.0 & \pm 5.9\end{aligned}$ \\
\hline $\begin{array}{l}\text { Vulva } \\
\text { (36) }\end{array}$ & $\begin{array}{l}\text { TVT } \\
\text { Fibroleiomioma } \\
\text { Lipoma } \\
\text { Fibrosarcoma } \\
\text { Leiomiosarcoma } \\
\text { Leiomioma } \\
\text { Hemangiosarcoma } \\
\text { Carcinoma escamoso } \\
\text { Plasmocitoma maligno } \\
\text { Mastocitoma }\end{array}$ & $\begin{array}{l}22 \\
3 \\
2 \\
2 \\
2 \\
1 \\
1 \\
1 \\
1 \\
1\end{array}$ & $\begin{aligned} 61.1 & \pm 15.9 \\
8.3 & \pm 9.0 \\
5.6 & \pm 7.5 \\
5.6 & \pm 7.5 \\
5.6 & \pm 7.5 \\
2.8 & \pm 5.4 \\
2.8 & \pm 5.4 \\
2.8 & \pm 5.4 \\
2.8 & \pm 5.4 \\
2.8 & \pm 5.4\end{aligned}$ \\
\hline & Total & 96 & \\
\hline
\end{tabular}



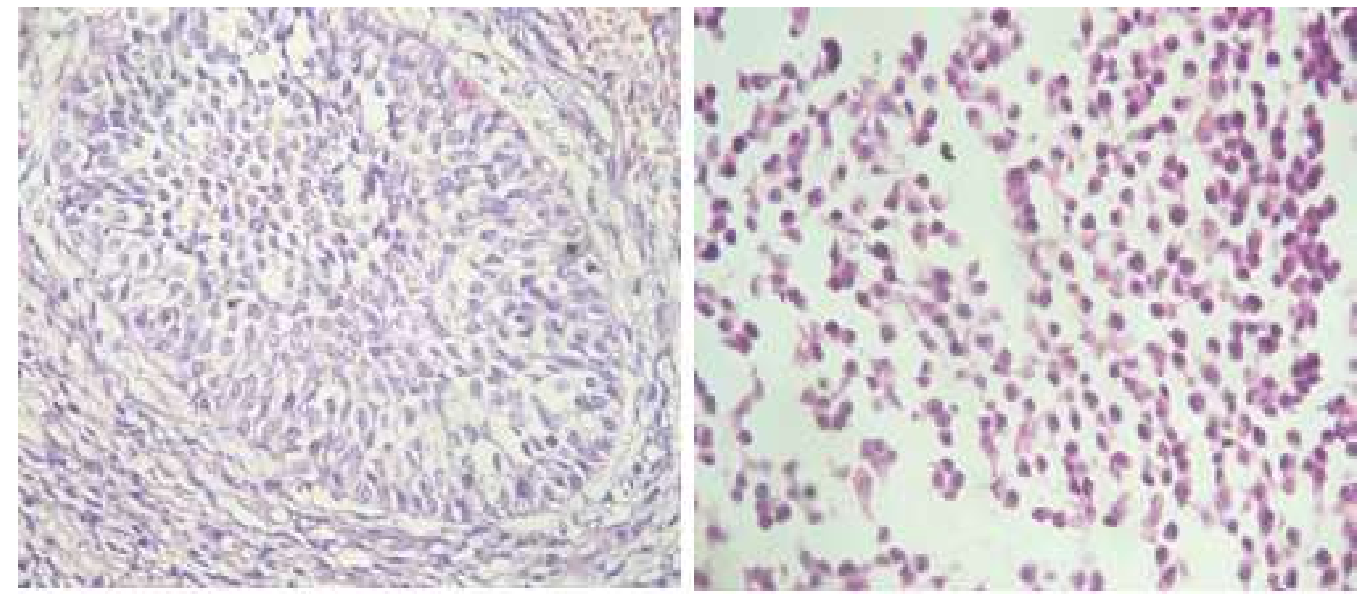

Figura 1. Microscopía de tumor de células de la granulosa (izquierda) y de tumor venéreo transmisible - TVT (derecha). Tinción H-E, 400X. Fuente: Archivo del Laboratorio de Histología, Embriología y Patología Animal (FMV-UNMSM)

las razas Bóxer y Labrador presentaron la mayor casuística ( $9 / 56$ en ambos casos). En el caso de felinos, las neoplasias se presentaron en dos pacientes de raza Persa y en uno cruzado.

Respecto al estrato etario, la mayor frecuencia se observó en canes de 8-12 años (43.75\%; 42/96) (Cuadro 1). En dos casos no se tuvo el registro de la edad. En el caso de los felinos, dos casos fueron del grupo etario mayor de 12 años y uno en el grupo de 8-12 años.

La neoplasia ovárica más frecuente en caninos fue el tumor de células de la granulosa (Figura 1) con $47.6 \pm 21.4 \% \mathrm{IC}_{95 \%}$, en tanto que en el útero fue el leiomioma $(50.0 \pm 40.0 \%$ $\mathrm{IC}_{95 \%}$ ), mientras que en vagina y vulva fue el tumor venéreo transmisible (TVT) (39.4 \pm 16.7 y $61.1 \pm 15.9 \% \mathrm{IC}_{95 \%}$, respectivamente (Cuadro 2).

En el caso de felinos, no se hallaron neoplasias vaginales ni vulvares. Las tres neoplasias correspondieron a dos casos de disgerminomas en ovario y un caso de fibroadenoma de forma polipoide en útero.

\section{Discusión}

La frecuencia de neoplasias del tracto reproductor femenino en caninos en relación al total de neoplasias en la especie representó el $5.99 \pm 1.16 \% \mathrm{IC}_{95 \%}(96 / 1603)$, valor menor al reportado por Clavo (1995) quién encontró un valor de $8.6 \%$ (46/534), aunque similar a los resultados de De Vivero (2009) con $6.0 \%(65 / 1092)$. En la especie felina, las neoplasias del tracto reproductor femenino representaron el $4.41 \pm 4.88 \% \mathrm{IC}_{95 \%}(3 / 68)$ frente al total de neoplasias, similar al reporte de Castro (2010) con 4.1\%. La baja frecuencia de este tipo de neoplasias podría atribuirse a que gran parte de la población canina y felina es esterilizada a una edad temprana.

Según la raza en caninos, la mayor frecuencia se encontró en los Bóxer. De Vivero (2009) señala que esta raza tiene una gran propensión a presentar procesos neoplásicos.

La mayor frecuencia de neoplasias reproductivas en el estrato etario de 8-12 años concuerda con el estudio de Clavo (1995) llevado a cabo con registros de este mismo la- 
boratorio. Asimismo, diversos autores (McEntee y Nielsen, 1976; Patnaik y Greenlee, 1987; Hermo et al., 2010), indican que las frecuencias de neoplasias del tracto reproductor femenino aumentan según la edad, posiblemente debido a la mayor exposición y desarrollo de las noxas causantes de la neoplasia. Por otro lado, las escasas observaciones en felinos indicaron la ocurrencia de estas neoplasias en animales mayores de 12 años, mientras que Gelberg y McEntee (1985) indican que la edad promedio de presentación es de 6.7 años.

La vulva y la vagina fueron las estructuras anatómicas más afectadas por neoplasias en caninos $(37.5 \pm 9.7 \%$ y $34.4 \pm$ $9.5 \%$, respectivamente), donde el TVT, neoplasia bastante común en el el país (Mendoza, 2006), fue la de mayor frecuencia. Las neoplasias ováricas $(21.9 \pm 8.3 \%)$ presentaron una mayor frecuencia que la reportada en el estudio de Clavo (2.2\%) en este laboratorio y en el de Gonzáles et al. (15.4\%) en Ciudad de México.

Dentro de las neoplasias estromales de células sexuales, el tumor de células de la granulosa mostró la mayor frecuencia frente al total de neoplasias ováricas (47.6 $\pm 21.4 \%$ ), lo cual concuerda con Gonzáles et al. (1997), quienes reportan una frecuencia de $44.4 \%$ dentro de las neoplasias ováricas.

Las neoplasias de origen epitelial y de células germinales mostraron una baja frecuencia, pese a que se reportan frecuencias de 40-50\% (Argyle, 2008; Thomson y Britt, 2012).

La menor frecuencia de neoplasias en útero frente al total de neoplasias del tracto reproductor, concuerda con los hallazgos de Clavo (6.5\%) y De Vivero (7.7\%) en el país, pero ligeramente menor a lo reportado por Gonzáles (9.4\%) en México. El leiomioma representó la neoplasia uterina canina más frecuente $(50.0 \pm 40.0 \%)$. Autores como Klein (2007), North y Banks (2009) y
Thomson y Britt (2012) concuerdan en que el leiomioma es la neoplasia más frecuente en útero canino, neoplasia que tiene su origen en las fibras musculares lisas del útero.

Dentro del grupo de neoplasias mesenquimales vaginales en las perras, las neoplasias fibrocíticas presentaron la mayor frecuencia $(21.2 \pm 14.0)$ frente al total de neoplasias vaginales, frecuencia bastante más grande que el $11.9 \%$ reportado por Clavo (1995).

\section{Conclusiones}

- La frecuencia de neoplasias del tracto reproductor de hembras caninos fue de $5.99 \pm 1.16 \% \mathrm{IC}_{95 \%}(96 / 1603)$ y para felinos fue de $4.41 \pm 4.88 \% \mathrm{IC}_{95 \%}(3 /$ 68 ), con base a los diagnósticos histopatológicos del Laboratorio de Patología Animal de la Universidad Nacional Mayor de San Marcos durante el periodo 2007-2015.

- En caninos, la mayor frecuencia se presentó en razas puras $(60.22 \pm 9.95 \%)$, en canes entre 8 a 12 años $(43.75 \pm$ $9.92 \%$ ) y mayormente en la zona vulvar $(37.50 \pm 9.68 \%)$.

- En caninos, la neoplasia más frecuente en el ovario fue el tumor de células de la granulosa $(47.6 \pm 21.4 \%)$, en útero fue el leiomioma $(50.0 \pm 40.0 \%)$ y en vulva $\mathrm{y}$ vagina fue el tumor venéreo transmisible $(39.4 \pm 16.7 \%)$.

- En felinos, se encontraron dos disgerminomas en ovarios y un fibroadenoma polipoide en útero.

\section{Literatura Citada}

1. Argyle D. 2008. Tumors of the reproductive tract. In: Argyle D, Brearley M, Turek M (eds). Decision making in small animal oncology. USA: WileyBlackwell. p 315-325. 
2. Baba A, Catoi C. 2007. Comparative oncology. Bucarest: Ed Academia Rumana. 548 p.

3. Castro T. 2010. Frecuencia de neoplasias en la especie felina diagnosticadas histopatológicamente en el Laboratorio de Histología, Embriología y Patología de la FMV-UNMSM Periodo 1996-2007. Tesis de Médico Veterinario. Lima: Univ Nacional Mayor de San Marcos. 54 p.

4. Clavo O. 1995. Neoplasias de los órganos genitales de la perra. Estudio estadístico año 1973-1994. Tesis de Médico Veterinario. Lima: Univ Nacional Mayor de San Marcos. 40 p.

5. Das U, Das AK. 2000. Review of canine transmissible venereal sarcoma. Vet Res Commun 24: 545-556.

6. De Vivero LE. 2009. Frecuencia de neoplasias caninas diagnosticadas en el Laboratorio de Histología, Embriología y Patología Veterinaria de la FMVUNMSM periodo 1995-2006. Tesis de Médico Veterinario. Lima: Univ Nacional Mayor de San Marcos. 63 p.

7. Dobson J, Lascelles D. 2010. BSAVA Manual of canine and feline oncology. $3^{\text {rd }}$ ed. UK: Wiley. 364 p.

8. Gelberg HB, McEntee K. 1986. Pathology of the canine and feline uterine tube. Vet Pathol 23: 770-775.

9. González G, Sánchez CA, Vélez M, de Buen N. 1997. Neoplasias en aparato reproductor en perras: estudio retrospectivo de 6 años. Vet Méx 28: 31-34.

10. Hermo, G Rimavicius F. Gonzales D. 2010. Tumores del aparato reproductor femenino en caninos y felinos (ovario y útero). [Internet]. Disponible en: http:// www.oncoveterinaria.com.ar/contenidos/archivos/file/Nueva\%20carpeta$\% 20$ prueba/CVPBA\%2046\%20 \%20Tumores.pdf

11. Jubb KVF, Kennedy PC. Palmer N. 1991. Patología de animales domésticos. $3^{\text {ra }}$ ed. Nueva York, EEUU: Hemisferio Sur. 2100 p.
12. Klein M. 2007. Tumors of the female reproductive system. In: Withrow, Mac Ewen's. Small animal clinical oncology. USA: Saunders Elsevier. p 610-618.

13. McEntee K, Nielsen S. 1976. Tumors of the ovary and tumors of the female genital tract. Bull World Health Organ 53: 203-226.

14. Medina I. 2015. Frecuencia de tumores en piel de caninos diagnosticados histopatológicamente en el Laboratorio de Histología, Embriología y Patología Veterinaria de la UNMSM durante el periodo 1999-2012. Tesis de Médico Veterinario. Lima: Univ Nacional Mayor de San Marcos. 86 p.

15. Mello Martins MI, Ferreiro de Souza F, Gobello C. 2005. The canine transmissible venereal tumor: etiology, pathology, diagnosis and treatment. [Internet]. Disponible en: http:// www.biologia.studies.uj.edu.pl/ $\sim$ joachimiak/WWW/Joachimiak/ ciekawostki/Canine\%20tumor.pdf

16. Mendoza N. 2006. Frecuencia del tumor venéreo transmisible en caninos: casuística del Laboratorio de Histología, Embriología y Patología Veterinaria-Facultad de Medicina Veterinaria - Universidad Nacional Mayor de San Marcos (periodo 1998- 2004). Tesis de Médico Veterinario. Lima: Univ Nacional Mayor de San Marcos. 43 p.

17. Miller MA, Ramos-Vara JA, Dickerson MF, Johnson GC, Pace LW, Kreeger JM, et al. 2003. Uterine neoplasia in 13 cats. J Vet Diagn Invest 15: 515-522.

18. Morris J, Dobson J. 2001. Small animal oncology. UK: Wiley-Blackwell. 298 p.

19. North S, Banks T. 2009. Introduction to small animal oncology. UK: Saunders Elsevier. $298 \mathrm{p}$.

20. Patnaik AK, Greenlee PG. 1987. Canine ovarian neoplasms: a clinicopathologic study of 71 cases including histology of 12 granulosa cell tumors. Vet Pathol 24: 509-514. 
21. Sforna M, Brachelente C, Lepri E, Mechelli L. 2003. Canine ovarian tumours: a retrospective study of 49 cases. Vet Res Commun 27: 359-361.
22. Thomson M, Britt T. 2012. Reproductive system. In: Kudnig T, Seguin B (eds). Vet Surgical Oncol. USA: Wiley-Blackwell. p 341-363. 